The Astronomical Journal, 121:2664-2672, 2001 May

(C) 2001. The American Astronomical Society. All rights reserved. Printed in U.S.A.

\title{
CO OBSERVATIONS OF NGC 2359: THE MOLECULAR CLOUDS REVISITED
}

\author{
C. E. CAPPA ${ }^{1,2}$ \\ Instituto Argentino de Radioastronomía, CC 5, 1894 Villa Elisa, Argentina; and Facultad de Ciencias Astronómicas y Geofísicas, \\ Universidad Nacional de La Plata, Paseo del Bosque s/n, 1900 La Plata, Argentina; ccappa@fcaglp.fcaglp.unlp.edu.ar \\ M. RuBIO ${ }^{2}$ \\ Departamento de Astronomía, Universidad de Chile, Casilla 36-D, Santiago, Chile; mrubio@das.uchile.cl \\ AND \\ W. M. Goss \\ National Radio Astronomy Observatory, P.O. Box 0, Socorro, NM 87801; mgoss@aoc.nrao.edu \\ Received 2000 December 5 ; accepted 2001 February 8
}

\begin{abstract}
Based on CO (2-1) observations obtained with the Swedish-ESO Submillimeter Telescope, the distribution of molecular material associated with the Wolf-Rayet ring nebula NGC 2359 has been determined. The angular resolution is $22^{\prime \prime}$, and the velocity resolution is $0.33 \mathrm{~km} \mathrm{~s}^{-1}$. Three molecular components are detected in the direction of the nebula. The bulk of the molecular gas is observed at $54 \mathrm{~km} \mathrm{~s}^{-1}$ and follows the southeastern border of the nebula. Adopting a distance $d=5 \mathrm{kpc}$ for NGC 2359, an $\mathrm{H}_{2}$ mass of about $1200 \pm 500 M_{\odot}$ appears to be related to the southern part of the nebula, while $140 \pm 50 M_{\odot}$ are linked to the filamentary windblown bubble. The volume density of the molecular gas related to the southern bar is $\geq 10^{3} \mathrm{H}_{2}$ molecules $\mathrm{cm}^{-3}$, while lower densities were estimated for the material associated with the filamentary windblown bubble. From the present data, it is not clear if the molecular gas at $37 \mathrm{~km} \mathrm{~s}^{-1}\left(\simeq 380 \pm 120 M_{\odot}, d=5 \mathrm{kpc}\right)$ is associated with the nebula, but the molecular material observed at $67 \mathrm{~km} \mathrm{~s}^{-1}\left(\simeq 70 \pm 25 M_{\odot}, d=5 \mathrm{kpc}\right)$ seems to be unconnected. The comparison between ionized, $\mathrm{H} \mathrm{I}$, and molecular distributions indicates that the $\mathrm{H}$ I filament detected with the Very Large Array is located at the interface between the ionized and molecular material, and that the $\mathrm{H}$ I filament at $54 \mathrm{~km} \mathrm{~s}^{-1}$ has originated in the photodissociation of the $\mathrm{H}_{2}$. Most of the molecular gas associated with the filamentary bubble seems to be interstellar in origin. The dynamics of the nebula is reanalyzed based on these new molecular results. It is consistent with either momentum conservation or an intermediate stage between energy and momentum conservation.
\end{abstract}

Key words: ISM: bubbles - ISM: molecules - ISM: individual (NGC 2359) stars: individual (HD 56925) - stars: Wolf-Rayet

\section{INTRODUCTION}

NGC 2359 is a ring nebula surrounding the Wolf-Rayet (W-R) star HD 56925 (W-R 7 in the catalog of van der Hucht et al. 1988). The WN 4b star (Smith et al. 1996) is located at $\alpha=7^{\mathrm{h}} 16^{\mathrm{m}} 10^{\mathrm{s}} 1, \delta=-13^{\circ} 7^{\prime} 31^{\prime \prime}(\mathrm{B} 1950.0 ; l=227.75, b=$ $-0.13)$. Figure 1 displays an $\mathrm{H} \alpha$ image of NGC 2359 as taken from Cappa et al. (1999, hereafter CGNO). The optical nebula, which has been discussed by Schneps et al. (1981, hereafter SHWB), consists of a filamentary shell of about 4.5 in size, a number of streamers of diffuse ionized gas and a southern bright region, the bar. The filamentary shell has been interpreted as a windblown bubble (WBB; Johnson \& Hogg 1965; Chu 1981).

CGNO presented radio continuum $(1465 \mathrm{MHz})$ and $\mathrm{H}$ I $21 \mathrm{~cm}$ line observations of the nebula carried out with the Very Large Array (VLA), with resolutions of $\simeq 30^{\prime \prime}$ and $\simeq 45^{\prime \prime}$, respectively. The radio continuum emission shows an excellent correlation with the nebular line emission. The ionized mass in the filamentary shell was estimated to be $\simeq 70 d_{5 \mathrm{kpc}}^{2} M_{\odot}\left(d_{5 \mathrm{kpc}}\right.$ is the distance in units of $\left.5 \mathrm{kpc}\right)$, indicating that the shell mainly consists of interstellar matter, while the ionized mass in the whole complex is in the range

\footnotetext{
${ }^{1}$ Member of Carrera del Investigador Científico, CONICET, Argentina.

${ }^{2}$ Visiting Astronomer, European Southern Observatory.
}

$900-1100 d_{5 \mathrm{kpc}}^{2} M_{\odot}$. The $\mathrm{H}$ I emission distribution revealed for the first time neutral gas associated with the optical ring nebula at both 54 and $63 \mathrm{~km} \mathrm{~s}^{-1}$. The $\mathrm{H}$ I component at $54 \mathrm{~km} \mathrm{~s}^{-1}$ consists of an arclike filament that closely borders the northern part of the optical northwestern streamer, the eastern region of the filamentary WBB, and the southern region of the bar (see Fig. 5 of CGNO). The $\mathrm{H}$ I emission at $63 \mathrm{~km} \mathrm{~s}^{-1}$ covers most of the nebula, with the brightest $\mathrm{H}$ I clumps defining an arclike structure that surrounds most of the WBB and the southern bar (see Fig. 6, CGNO). (Throughout this paper, radial velocities are given with respect to the LSR.)

Molecular observations in the direction of NGC 2359 were first obtained by Schneps et al. (1981) with the $12 \mathrm{~m}$ NRAO telescope at Kitt Peak (angular resolution $=66^{\prime \prime}$ ). These CO (1-0) observations revealed molecular line emission at 37, 54, and $67 \mathrm{~km} \mathrm{~s}^{-1}$ (see Fig. 1, Schneps et al. 1981). The molecular cloud at $54 \mathrm{~km} \mathrm{~s}^{-1}$ is clearly interacting with the nebula, while the cloud at $37 \mathrm{~km} \mathrm{~s}^{-1}$ might also be associated. The derived $\mathrm{H}_{2}$ masses in the concentrations at 37 and $54 \mathrm{~km} \mathrm{~s}^{-1}$ were $800 d_{5 \mathrm{kpc}}^{2}$ and $90 d_{5 \mathrm{kpc}}^{2} M_{\odot}$, respectively. The region was also recently reobserved using the same telescope by Rizzo, Martin-Pintado, \& Mangum (2001), who estimated $96 d_{5 \mathrm{kpc}}^{2} M_{\odot}$ and $300 d_{5 \mathrm{kpc}}^{2} M_{\odot}$ for the components at 37 and $54 \mathrm{~km} \mathrm{~s}^{-1}$. St-Louis et al. (1998) found excited $\mathrm{H}_{2}$ in the southern bar.

The comparison of the optical emission with the $\mathrm{H} \mathrm{I}$ emission at $54 \mathrm{~km} \mathrm{~s}^{-1}$ and the molecular emissions at 54 and 


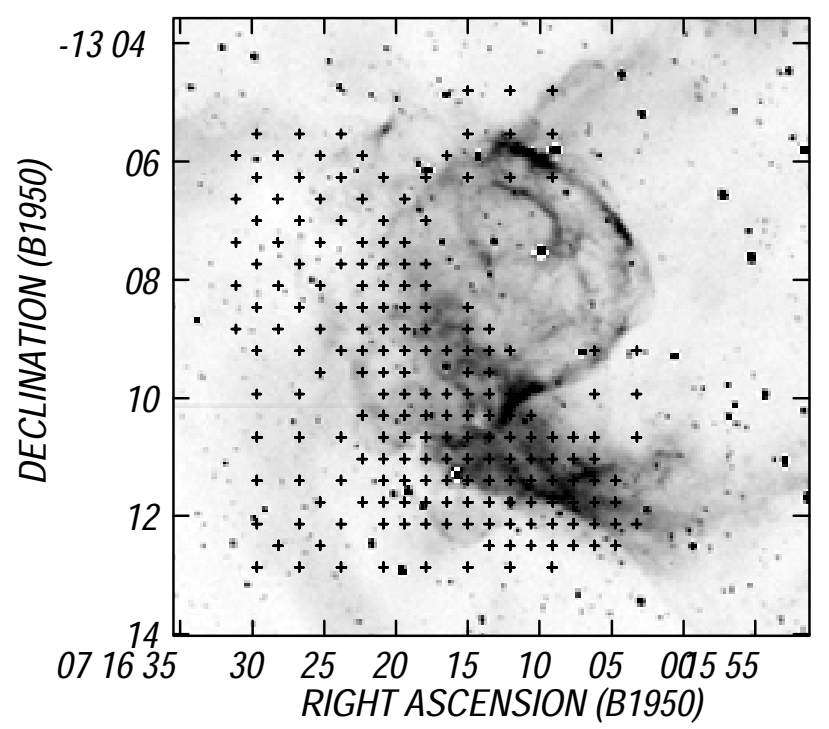

Fig. 1.-Narrowband $\mathrm{H} \alpha$ image of NGC 2359 taken from CGNO. The gray scale is in arbitrary units. The plus signs mark the position of the 197 points observed in $\mathrm{CO}$ lines.

$37 \mathrm{~km} \mathrm{~s}^{-1}$ suggests that the $\mathrm{H} \mathrm{I}$ emission at $54 \mathrm{~km} \mathrm{~s}^{-1}$ marks the location of the ionization fronts (CGNO), in agreement with the $[\mathrm{N} \mathrm{II}]$ emission (SHWB).

Molecular observations in the direction of other W-R ring nebulae were performed with the Swedish-ESO Submillimeter Telescope (SEST) by Marston et al. (1999a, 1999b). They have found molecular gas associated with the ring nebulae around W-R 16 and 75 (RCW 104), which has probably been ejected in previous evolutionary phases of the W-R stars.

In this paper, we present $\mathrm{CO}(2-1)$ and new $\mathrm{CO}(1-0)$ observations in the direction of NGC 2359, carried out with the $15 \mathrm{~m}$ SEST at La Silla, Chile. Our aim is to investigate in detail the distribution and characteristics of the molecular material associated with NGC 2359, to compare the distributions of the various gas components, and to analyze the interaction between the ionized and neutral gas with the molecular material. Kinetic efficiencies in windblown bubbles depend on the stellar wind parameters, on the expansion velocity of the bubbles, and on the amount of material that participates in the expansion (see Treffers \& Chu 1982). Thus, reliable $\mathrm{H}_{2}$ masses related to the complex can provide valuable information about the energetic properties of NGC 2359.

\section{OBSERVATIONS AND DATA REDUCTION}

We observed the ${ }^{12} \mathrm{CO}(J=1 \rightarrow 0)(115 \mathrm{GHz})$ and ${ }^{12} \mathrm{CO}$ $(J=2 \rightarrow 1)(230 \mathrm{GHz})$ rotational lines during 2000 January 11-16 with the $15 \mathrm{~m}$ SEST located at La Silla, Chile. The telescope and receivers are described in detail by Booth et al. (1989). The half-power beamwidth (HPBW) of the telescope was $44^{\prime \prime}$ and $22^{\prime \prime}$ at 115 and $230 \mathrm{GHz}$, respectively. The high-resolution acousto-optical spectrometer had a total bandwidth of about $100 \mathrm{MHz}$ and a resolution of 80 $\mathrm{KHz}$, corresponding to velocity resolutions of $\simeq 0.209 \mathrm{~km}$ $\mathrm{s}^{-1}$ at $115 \mathrm{GHz}\left[\mathrm{CO}(1-0)\right.$ line] and $\simeq 0.104 \mathrm{~km} \mathrm{~s}^{-1}$ at 230 $\mathrm{GHz}[\mathrm{CO}(2-1)$ line $]$. The observed velocity interval ranged from 23 to $76 \mathrm{~km} \mathrm{~s}^{-1}$ at $230 \mathrm{GHz}$ and from -4 to $104 \mathrm{~km}$ $\mathrm{s}^{-1}$ at $115 \mathrm{GHz}$. The system temperatures were $\simeq 200 \mathrm{~K}$ at
$230 \mathrm{GHz}$ and $\simeq 300 \mathrm{~K}$ at $115 \mathrm{GHz}$. The pointing was checked once during each observing run on the $\mathrm{SiO}(v=1$, $J=2 \rightarrow 1$ ) maser source Ori A. The pointing errors were $3^{\prime \prime}$. The uncertainty in the intensity calibration was $10 \%$.

The ${ }^{12} \mathrm{CO}(2-1)$ and ${ }^{12} \mathrm{CO}(1-0)$ lines were acquired simultaneously in the position-switching mode on a grid with a spacing of $22^{\prime \prime}$. The off-source position was $\alpha=$ $7^{\mathrm{h}} 16^{\mathrm{m}} 4.2, \delta=-13^{\mathrm{s}} 7^{\prime} 48^{\prime \prime}(\mathrm{B} 1950.0)$. The ${ }^{12} \mathrm{CO}$ observations were taken of 197 points, which are indicated by plus signs in Figure 1. The ${ }^{13} \mathrm{CO}(2-1)$ and ${ }^{13} \mathrm{CO}(1-0)$ observations were carried out at four positions within the observed area, at the strongest $\mathrm{CO}$ peaks. The observed line intensities were expressed as main-beam brightness temperatures $T_{\mathrm{mb}}$, by dividing the antenna temperature $T_{A}^{*}$ by the main-beam efficiency $\eta_{\mathrm{mb}}$, equal to 0.70 and 0.50 at 115 and $230 \mathrm{GHz}$, respectively.

The spectra were reduced using the CLASS software (GILDAS working group). After smoothing the ${ }^{12} \mathrm{CO}$ profiles to a velocity resolution of 0.43 and $0.33 \mathrm{~km} \mathrm{~s}^{-1}$ at 115 and $230 \mathrm{GHz}$, respectively, the typical rms noise temperature was $0.15 \mathrm{~K}\left(T_{\mathrm{mb}}\right)$ at $230 \mathrm{GHz}$ and $0.22 \mathrm{~K}$ at 115 $\mathrm{GHz}$, after an integration time of 2 minutes. The rms noise temperature of the ${ }^{13} \mathrm{CO}(2-1)$ spectra was $0.20 \mathrm{~K}$ and $0.10 \mathrm{~K}$ for ${ }^{13} \mathrm{CO}(1-0)$, after an integration time of 6 minutes.

\section{RESULTS AND DISCUSSION}

The present high-sensitivity and high angular resolution observations enabled us to map in detail the molecular material in the environs of NGC 2359. The emission appears concentrated at 37,54 , and $67 \mathrm{~km} \mathrm{~s}^{-1}$, as previously found by SHWB. The main results of each velocity component are discussed separately in the following sections. Since the $J=1 \rightarrow 0$ data reveal a structure similar to that observed in the (2-1) line, but with a lower angular resolution and higher rms noise temperature, our analysis will focus on the (2-1) observations.

The distance to the nebula is a matter of some debate. Van der Hucht (2001) derived a spectrophotometric distance $d_{*}=3.7 \mathrm{kpc}$ for the W-R star, in agreement with previous determinations by van der Hucht et al. (1988) and Conti \& Vacca (1990). Assuming an uncertainty of \pm 0.5 mag in the absolute magnitude, the error in the photometric distance is $\pm 0.9 \mathrm{kpc}$. The LSR systemic velocity of NGC 2359 is $54 \mathrm{~km} \mathrm{~s}^{-1}$ (see CGNO, and references therein). The analytical fit to the flat galactic rotation curve by Brand \& Blitz (1993) predicts a kinematical distance $d_{k} \simeq 5.9 \pm 1.0$ $\mathrm{kpc}$ for gas at this velocity, where the uncertainty in $d_{k}$ originates in the presence of noncircular motions of about $\pm 7 \mathrm{~km} \mathrm{~s}^{-1}$ (Burton 1988). A kinematic distance $d_{k} \simeq 6 \mathrm{kpc}$ is also derived from Figures $2 a$ and $2 b$ of Brand \& Blitz (1993), which include the observed noncircular motions. Consequently, we adopted a mean distance $d=5.0 \pm 1.3$ $\mathrm{kpc}$, which takes into account both the photometric and the kinematic distances. A distance scaling factor is indicated in our results to facilitate the conversion to other distances.

\subsection{Molecular Cloud at $54 \mathrm{~km} \mathrm{~s}^{-1}$ 3.1.1. CO Distribution}

The $\mathrm{CO}$ emission distribution obtained from the ${ }^{12} \mathrm{CO}$ (2-1) data is displayed in Figures 2 and 3. Figure 2 shows an overlay of an $\mathrm{H} \alpha$ image of NGC 2359 and the ${ }^{12} \mathrm{CO}$ integrated emission $\int T_{\mathrm{mb}} d v\left(\mathrm{~K} \mathrm{~km} \mathrm{~s}^{-1}\right)$ within the velocity interval $47.6-56.5 \mathrm{~km} \mathrm{~s}^{-1}$, where the $54 \mathrm{~km} \mathrm{~s}^{-1}$ component 


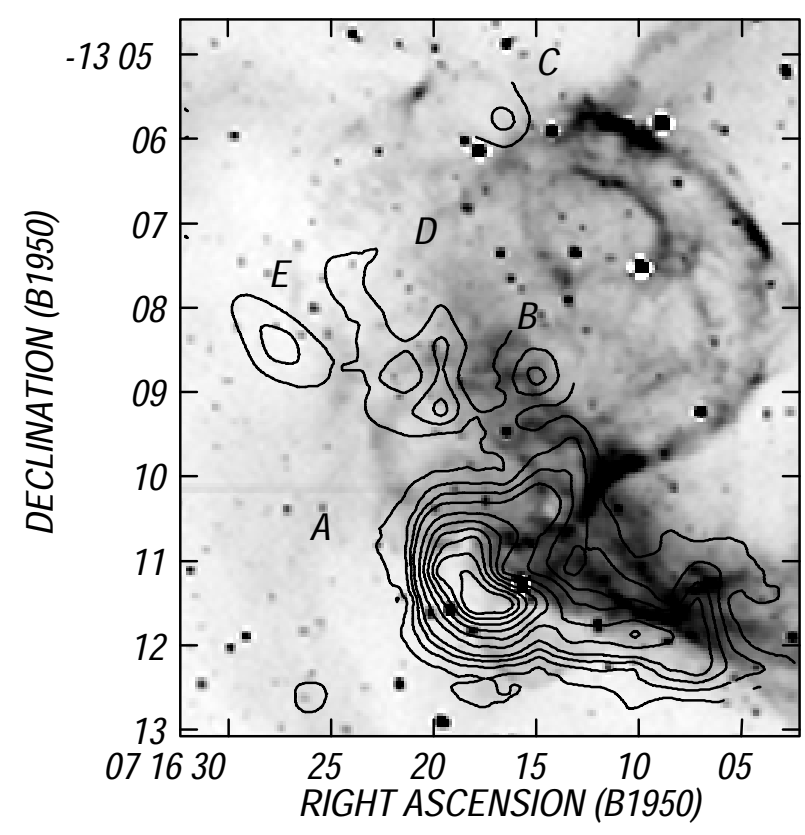

FIG. 2.-Integrated CO (2-1) line emission for the component at $54 \mathrm{~km}$ $\mathrm{s}^{-1}$ superposed on the $\mathrm{H} \alpha$ image of the nebula. The contour lines are 2.4, $4.8,7.2,9.6,14.4,19.2,24.0,28.8,33.6$, and $38.4 \mathrm{~K} \mathrm{~km} \mathrm{~s}^{-1}$.

was detected. Figure 3 displays a series of ${ }^{12} \mathrm{CO}(2-1)$ images showing $T_{\mathrm{mb}}$ within the velocity range $46.0-59.0 \mathrm{~km}$ $\mathrm{s}^{-1}$ in intervals of $1.6 \mathrm{~km} \mathrm{~s}^{-1}$. To facilitate the comparison with the ionized gas, the first panel of Figure 3 shows the optical image of the nebula. The plus signs in the last panel mark the position of the 197 points observed in ${ }^{12} \mathrm{CO}$ lines. Most of the $\mathrm{CO}$ emission appears concentrated in a structure that encircles the southern and eastern portions of the bar, named cloud A (Fig. 2). A number of smaller CO clouds were also detected at $\delta>-13^{\circ} 10^{\prime}$ (B1950.0). They are indicated as $\mathrm{B}, \mathrm{C}, \mathrm{D}$, and $\mathrm{E}$ in the same figure.

Cloud A consists of two cores (Fig. 2). The brightest clump is centered at $\alpha=7^{\mathrm{h}} 16^{\mathrm{m}} 18^{\mathrm{s}}, \delta=-13^{\circ} 11^{\prime} .5$ (B1950.0; named $\mathrm{E}$ clump), while the smaller one is observed at $\alpha=7^{\mathrm{h}} 16^{\mathrm{m}} 10^{\mathrm{s}}, \delta=-13^{\circ} 11^{\prime} 8$ (B1950.0; named W clump). The comparison of the molecular and the optical emissions shows that the strongest $\mathrm{CO}$ emission in cloud $\mathrm{A}$ follows the sharp southern, eastern, and northeastern borders of the bar, encircling the brightest optical emission regions. Strong optical absorption is observed at the position of the $\mathrm{E}$ clump. The weak optical emission region at $\alpha=7^{\mathrm{h}} 16^{\mathrm{m}} 15^{\mathrm{s}}$, $\delta=-13^{\circ} 12^{\prime} .2$ (B1950.0; see also Fig. 7, in which the contrast facilitates the identification of this region) matches the outer southern border of the brightest $\mathrm{CO}$ emission. $\mathrm{E}$ clump extends to the northwest, delineating the bright optical filament at $\alpha=7^{\mathrm{h}} 16^{\mathrm{m}} 11^{\mathrm{s}} .5, \delta=-13^{\circ} 10^{\prime}$ (B1950.0), and continues to the north, where it overlaps the filamentary shell. W clump delineates the southern bar near $\alpha=7^{\mathrm{h}} 16^{\mathrm{m}} 10^{\mathrm{s}}, \delta=-13^{\circ} 11^{\prime} 7$ (B1950.0). This emission extends slightly to the north at $\alpha=7^{\mathrm{h}} 16^{\mathrm{m}} 7^{\mathrm{s}}$ (B1950.0), where the bright optical filament ends. W clump is more easily identified in Figure 3 within the velocity range $49.2 \leq v \leq 54.1$ $\mathrm{km} \mathrm{s}^{-1}$. The gap at $\alpha=7^{\mathrm{h}} 16^{\mathrm{m}} 13^{\mathrm{s}}, \delta=-13^{\circ} 11^{\prime} 7$ (B1950.0) between both the E and W clumps is clearly observed in Figure 3 for $v \leq 54.1 \mathrm{~km} \mathrm{~s}^{-1}$. At larger velocities a small clump is detected at this position.
Cloud B overlaps the filamentary shell, while clouds D and $\mathrm{E}$ lie toward the east, with cloud $\mathrm{D}$ located just outside the filamentary bubble. Cloud $\mathrm{E}$ is located in a region where strong optical absorption is present. Cloud $\mathrm{C}$ is observed to the north, close to the border of the filamentary bubble.

The comparison of the optical and molecular emissions suggests that the molecular gas is being compressed by ionization and/or shock fronts and is clearly interacting with the optical nebula. As pointed out above, the molecular material delineates the position of the ionization fronts, which are seen edge-on.

A Gaussian fit to the ${ }^{12} \mathrm{CO}(2-1)$ spectra indicates that the mean LSR velocity of this component ranges from 52.0 $54.6 \mathrm{~km} \mathrm{~s}^{-1}$. The lines are broad (see Fig. $4 a-4 d$ ), with full widths at half-maximum (FWHMs) in the range of 2.5-5.7 $\mathrm{km} \mathrm{s}^{-1}$, slightly larger than the values obtained by SHWB. Most of the broader profiles correspond to cloud A.

The Gaussian analysis shows a single gas component with a velocity close to $54 \mathrm{~km} \mathrm{~s}^{-1}$. Only two profiles indicate the presence of an additional weak molecular component with a mean velocity of about $46 \mathrm{~km} \mathrm{~s}^{-1}$ (peak $T_{m b} \simeq 0.7 \mathrm{~K}$ ). These profiles correspond to Cloud $\mathrm{D}$ and are shown in Fig. $4 c$ and $4 d$. The spectra were obtained in direction of the borders of a small ringlike optical structure observed to the southeast of the main filamentary bubble. An inspection of the $\mathrm{H}$ I emission distribution at $v=46.9$ $\mathrm{km} \mathrm{s}^{-1}$ obtained with the VLA (see Fig. 4 in CGNO) shows the presence of an $\mathrm{H}$ I cloud at $\alpha=7^{\mathrm{h}} 16^{\mathrm{m}} 20^{\mathrm{s}}, \delta=-13^{\circ} 9^{\prime} .5$ (B1950.0), which overlaps the inner region and the borders of the small optical shell described above. We interpret this material to be the $\mathrm{H}$ I counterpart of the weak molecular component at $46 \mathrm{~km} \mathrm{~s}^{-1}$. Goudis et al. (1983) also found ionized gas at a similar velocity in projection onto this small $\mathrm{H} \alpha$ feature.

Peak optical depths were derived for three positions in the direction of the $\mathrm{E}$ clump (cloud $\mathrm{A}$ ) for which ${ }^{13} \mathrm{CO}$ profiles were observed (at the coordinates $\alpha=7^{\mathrm{h}} 16^{\mathrm{m}} 16^{\mathrm{s}} .5$, $\delta=-13^{\circ} 11^{\prime} 24^{\prime \prime} ; \alpha=7^{\mathrm{h}} 16^{\mathrm{m}} 17^{\mathrm{s}} \cdot 9, \delta=-13^{\circ} 11^{\prime} 24^{\prime \prime} ;$ and $\alpha=$ $7^{\mathrm{h}} 16^{\mathrm{m}} 19^{\mathrm{s}} 4, \delta=-13^{\circ} 11^{\prime} 2^{\prime \prime}$ [B1950.0]). Two of these profiles are shown in Figures $4 a$ and $4 b$. The peak optical depth of the ${ }^{13} \mathrm{CO}$ line was estimated from the peak $T_{\mathrm{mb}}$ values as

$$
\tau\left({ }^{13} \mathrm{CO}\right) \simeq-\ln \left[1-T_{\mathrm{mb}}\left({ }^{13} \mathrm{CO}\right) /\left(10.58 J T_{\mathrm{exc}}-0.21\right)\right],
$$

where $J T_{\text {exc }}=\left(e^{10.58 / T_{\text {exc }}}-1\right)^{-1}$. The excitation temperature $T_{\text {exc }}$ was derived from the ${ }^{12} \mathrm{CO}$ line, assuming LTE and that ${ }^{12} \mathrm{CO}$ is optically thick. The peak optical depth of the ${ }^{12} \mathrm{CO}$ line was obtained as

$$
\begin{aligned}
\tau\left({ }^{12} \mathrm{CO}\right)= & {\left[v\left({ }^{13} \mathrm{CO}\right) / v\left({ }^{12} \mathrm{CO}\right)\right]^{2} } \\
& \times\left[\Delta v\left({ }^{13} \mathrm{CO}\right) / \Delta v\left({ }^{12} \mathrm{CO}\right)\right]\left({ }^{12} \mathrm{CO} /{ }^{13} \mathrm{CO}\right) \tau\left({ }^{13} \mathrm{CO}\right),
\end{aligned}
$$

where $v$ is the frequency of each transition, $\Delta v$ is the FWHM of the observed line and ${ }^{12} \mathrm{CO} /{ }^{13} \mathrm{CO}$ is the isotope ratio. Here the $T_{\text {exc }}$ 's in these regions, as derived from (2-1) lines, are about $14 \mathrm{~K}$. A ${ }^{12} \mathrm{CO} /{ }^{13} \mathrm{CO}$ isotope ratio of 70 was adopted (Wilson \& Matteucci 1992). Here $\tau\left({ }^{13} \mathrm{CO}\right) \simeq 0.2$ and $\tau\left({ }^{12} \mathrm{CO}\right) \simeq 7-9$ were estimated for the $(2-1)$ lines, while similar values were derived for the (1-0) lines. The ratio $W\left({ }^{12} \mathrm{CO}\right) / W\left({ }^{13} \mathrm{CO}\right) \quad\left[=\int T_{\mathrm{mb}}\left({ }^{12} \mathrm{CO}\right) d v / \int T_{\mathrm{mb}}\left({ }^{13} \mathrm{CO}\right) d v\right]$ for the three observed points is in the range 8-11 for both the $(2-1)$ and the $(1-0)$ lines.

The ${ }^{12} \mathrm{CO}(2-1) /{ }^{12} \mathrm{CO}(1-0)$ intensity ratio, which depends on the kinetic temperature, on the optical depth, and on the density, may provide information on the $\mathrm{CO}$ 

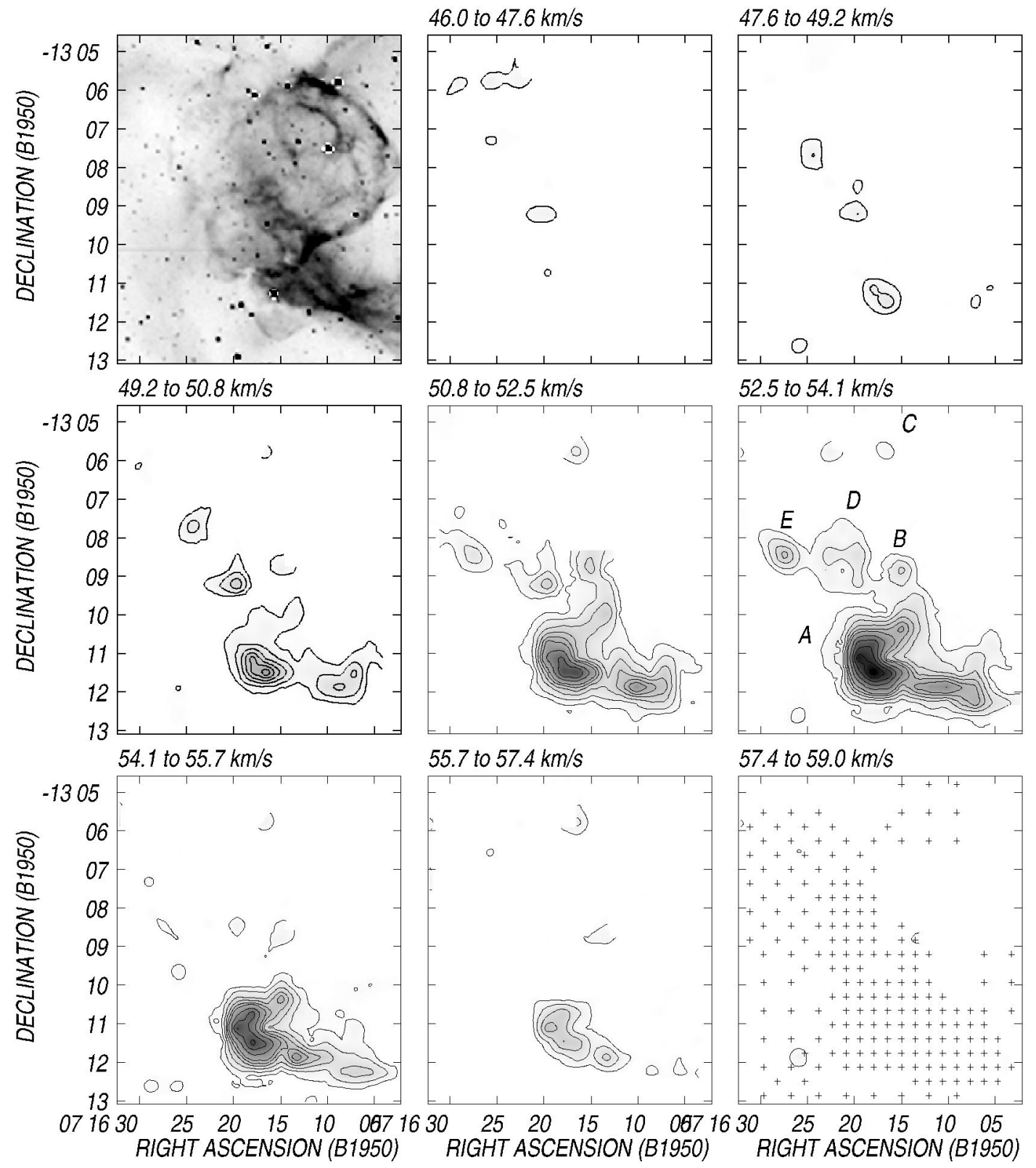

\section{7 to $57.4 \mathrm{~km} / \mathrm{s}$}

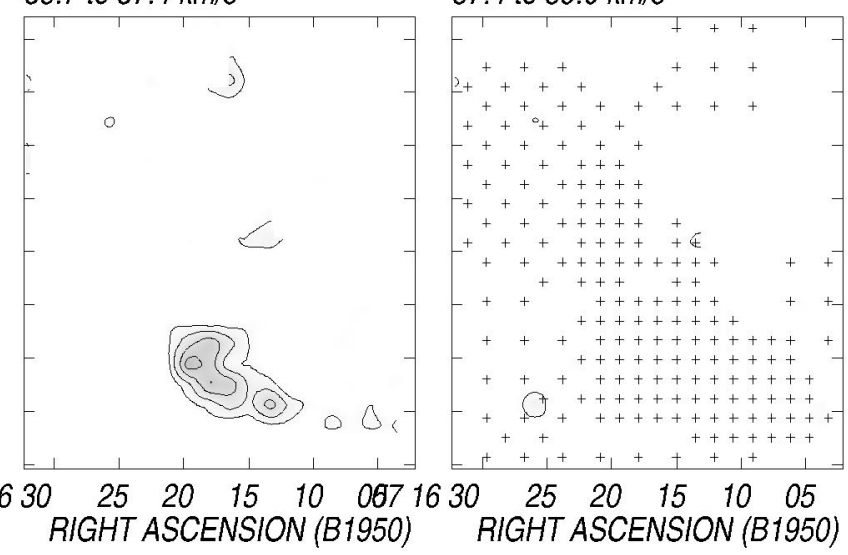

FIG. 3.-Images of $T_{\mathrm{mb}}$ within the LSR velocity range of $46.0-59.0 \mathrm{~km} \mathrm{~s}^{-1}$ in intervals of $1.6 \mathrm{~km} \mathrm{~s}^{-1}$. The first panel displays the H $\alpha$ image of the nebula. The gray scale of the $\mathrm{CO}$ images corresponds to $0.2-9.6 \mathrm{~K}$, while the contour lines are $0.42,0.84,1.4,2.0,2.6,3.2,4.4,5.6,6.8,8.0,9.2$, and $10.4 \mathrm{~K}$. The velocity interval of each image is indicated in the top left corner. The plus signs in the last image mark the position of the observed points.

excitation conditions. The ratio $R_{21}=\int T_{\mathrm{mb}}(2-1) d v /$ $\int T_{\mathrm{mb}}(1-0) d v$ obtained from the ${ }^{12} \mathrm{CO}$ data [after convolving the ${ }^{12} \mathrm{CO}(2-1)$ data to the angular resolution of the ${ }^{12} \mathrm{CO}(1-0)$ data] varies between 0.6 and 1.8 in cloud $\mathrm{A}$. Here $R_{21} \simeq 1.0 \pm 0.2$ is exhibited by the brightest parts of $\mathrm{E}$ clump, while $R_{21} \simeq 1.4 \pm 0.1$ was derived for W clump and $\simeq 1.3 \pm 0.7$ for the $\mathrm{CO}$ gas projected onto the bar. The largest value (1.9) was derived for cloud B, which is projected onto the main bubble.

The observed variation in the $R_{21}$ values can be understood in terms of changes in the excitation conditions of the molecular gas according to its location. The lower $R_{21}$ values are obtained toward E clump and the peripheral regions to the south and southeast of the clump, while $R_{21}>1.2$ is derived toward the bar and the W clump. The larger values may result if the clouds are dense, warm (kinetic temperature $\left.T_{K}>50 \mathrm{~K}\right)$, and less opaque $\left(\tau\left[{ }^{12} \mathrm{CO}\right.\right.$ $(2-1)]<$ <; see, e.g., Koo 1999; Sakamoto et al. 1994). Our results are consistent with the molecular material projected onto the bar and the filamentary shell, and in the W clump being exposed to a strong UV radiation field.

Examination of large-scale velocity gradient (LVG) diagrams (see, e.g., Castets et al. 1990; Sakamoto et al. 1994) indicates that in the optically thin case, $R_{21}>1$, requires densities larger than $10^{3} \mathrm{H}_{2}$ molecules $\mathrm{cm}^{-3}$ for kinetic temperatures in the range $20-50 \mathrm{~K}$. Higher densities should be expected in the optically thick case. Kinetic temperatures $\geq 30 \mathrm{~K}$ were found in photodissociation regions (PDRs) close to H II regions (see, e.g., Koo 1999; Castets et al. 1990).

Table 1 summarizes the central positions, dimensions, peak temperatures $T_{\mathrm{mb}}$, mean $\mathrm{H}_{2}$ column densities $N_{\mathrm{H}_{2}}, \mathrm{CO}$ luminosities $L_{\mathrm{CO}}$, molecular masses, and rough volume den- 

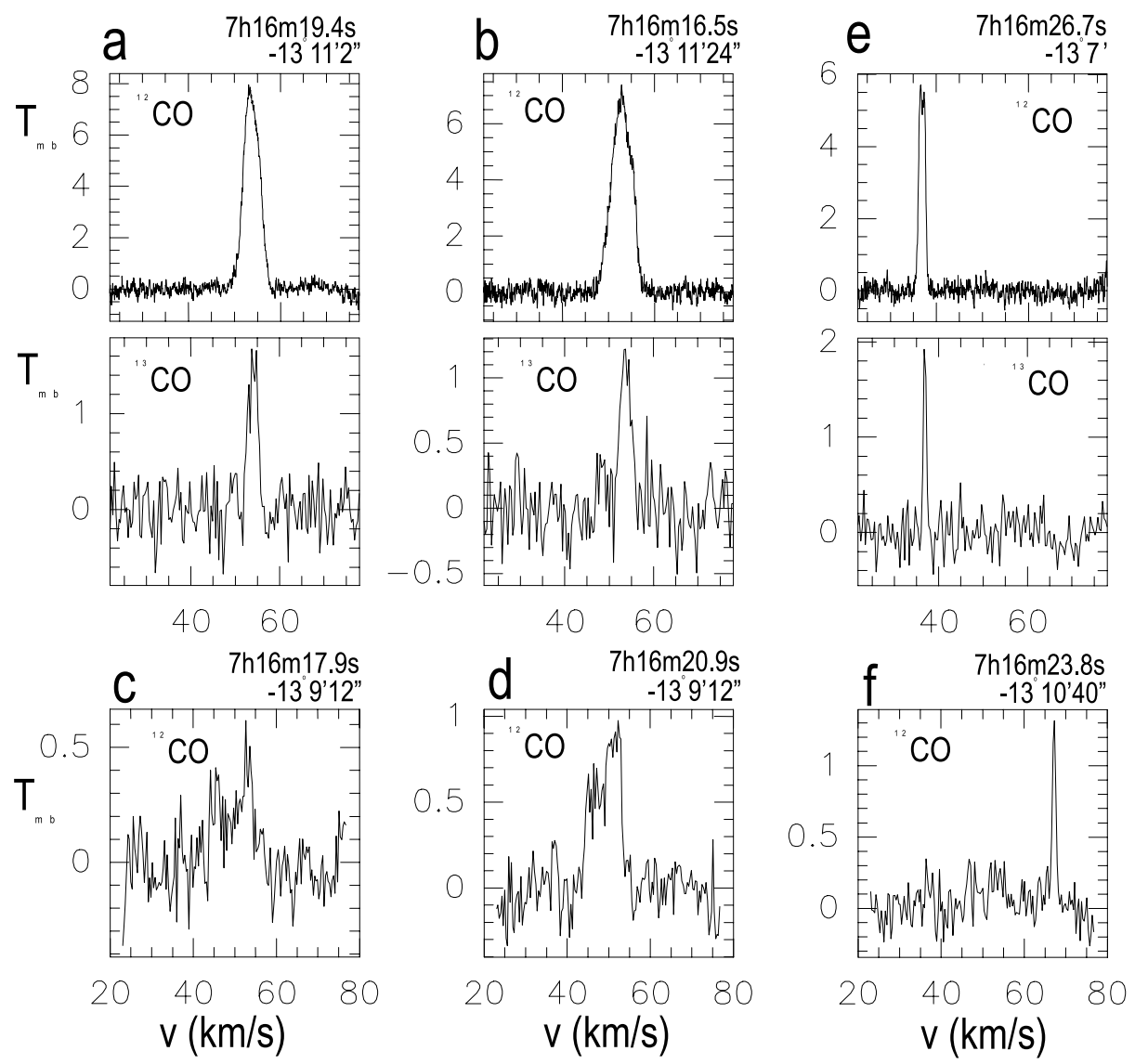

FIG. 4. - CO (2-1) spectra toward the three molecular clouds obtained with the SEST. The coordinates of each spectrum are indicated in the top right corner. $(a-b){ }^{12} \mathrm{CO}$ and ${ }^{13} \mathrm{CO}$ profiles obtained in the direction of the cloud at $54 \mathrm{~km} \mathrm{~s}^{-1} .(c-d){ }^{12} \mathrm{CO}$ profiles showing the weak molecular component at 46 $\mathrm{km} \mathrm{s}^{-1}$ (see $\S 3$ ). $(e)^{12} \mathrm{CO}$ and ${ }^{13} \mathrm{CO}$ profiles obtained in the direction of the cloud at $37 \mathrm{~km} \mathrm{~s}^{-1} .(f){ }^{12} \mathrm{CO}$ profile corresponding to the cloud at $67 \mathrm{~km} \mathrm{~s}^{-1}$.

sities $n$ of the individual clouds. The $\mathrm{H}_{2}$ column densities were derived from ${ }^{12} \mathrm{CO}(2-1)$ data by making use of the empirical relation between $W_{\mathrm{CO}}\left(=\int T_{\mathrm{mb}} d v\right)$ and $N_{\mathrm{H}_{2}}$. We adopted $N_{\mathrm{H}_{2}}=(2.5 \pm 0.9) W_{\mathrm{CO}} \times 10^{20} \mathrm{~cm}^{-2}\left(\mathrm{~K} \mathrm{~km} \mathrm{~s}^{-1}\right)^{-1}$, obtained from $\gamma$-ray studies of molecular clouds in the outer Galaxy (Digel et al. 1996). The CO luminosities are defined as $L_{\mathrm{CO}}=W_{\mathrm{CO}} A\left(\mathrm{~K} \mathrm{~km} \mathrm{~s}^{-1} \mathrm{pc}^{2}\right)$, where $A$ is the area in $\mathrm{pc}^{2}$. The masses include a Helium abundance of $10 \%$. Volume

TABLE 1

Molecular Clouds at $54 \mathrm{KM} \mathrm{s}^{-1}$

\begin{tabular}{|c|c|c|c|c|c|}
\hline Parameters & A & B & $\mathrm{C}$ & $\mathrm{D}$ & $\mathrm{E}$ \\
\hline \multicolumn{6}{|l|}{ Center position: } \\
\hline 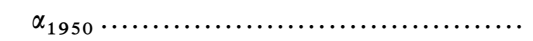 & 71613 & 71615 & 71617 & 71620 & 71628 \\
\hline 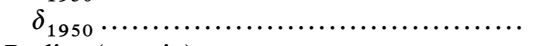 & -1311.5 & -138.7 & -135.7 & -138.7 & -138.5 \\
\hline Radius (arcmin) ......................... & $\ldots$ & 0.35 & 0.25 & 0.5 & $0.6 \times 0.3$ \\
\hline 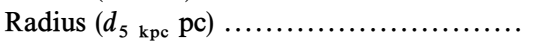 & $\ldots$ & 0.45 & 0.33 & 0.65 & $0.8 \times 0.4$ \\
\hline Peak temperature $T_{\mathrm{mb}}[\mathrm{CO}(2-1)](\mathrm{K}) \ldots \ldots$ & 11.6 & 1.8 & 1.2 & 2.4 & 1.1 \\
\hline $\begin{array}{l}\text { Mean } N_{\mathrm{H} 2}\left(10^{20} \mathrm{~cm}^{-2}\right) \ldots \ldots \ldots \ldots \ldots \ldots \ldots \ldots \ldots \ldots \ldots \ldots \\
d=5 \mathrm{kpc}:\end{array}$ & $26 \pm 10$ & $11 \pm 4$ & $10 \pm 4$ & $8 \pm 4$ & $10 \pm 4$ \\
\hline$L_{\mathrm{CO}}\left(\mathrm{K} \mathrm{km} \mathrm{s}^{-1} \mathrm{pc}^{2}\right) \ldots \ldots \ldots \ldots \ldots \ldots \ldots \ldots \ldots \ldots \ldots$ & 216.8 & 6.4 & 2.2 & 10.3 & 6.2 \\
\hline $\mathrm{H}_{2}$ mass $\left(M_{\odot}\right) \ldots \ldots \ldots \ldots \ldots \ldots$ & $1200 \pm 500$ & $35 \pm 10$ & $12 \pm 3$ & $55 \pm 20$ & $35 \pm 10$ \\
\hline $\begin{array}{l}n\left(10^{3} \mathrm{H}_{2} \text { molecules } \mathrm{cm}^{-3}\right) \ldots \ldots \ldots \ldots \ldots \\
d=3.7 \mathrm{kpc}:\end{array}$ & $5-10$ & $1-2$ & $1.0-2.0$ & $0.5-1$ & $0.5-1$ \\
\hline$L_{\mathrm{CO}}\left(\mathrm{K} \mathrm{km} \mathrm{s}^{-1} \mathrm{pc}^{2}\right) \ldots \ldots \ldots \ldots \ldots \ldots \ldots$ & 118.7 & 3.5 & 1.2 & 5.6 & 3.4 \\
\hline $\mathrm{H}_{2}$ mass $\left(M_{\odot}\right) \ldots \ldots \ldots \ldots \ldots \ldots \ldots \ldots \ldots \ldots \ldots \ldots$ & $660 \pm 260$ & $18 \pm 6$ & $6 \pm 2$ & $30 \pm 11$ & $18 \pm 6$ \\
\hline \multicolumn{6}{|l|}{$d=6.3 \mathrm{kpc}:$} \\
\hline$L_{\mathrm{CO}}\left(\mathrm{K} \mathrm{km} \mathrm{s}^{-1} \mathrm{pc}^{2}\right) \ldots \ldots \ldots$ & 344.2 & 10.2 & 3.5 & 16.4 & 9.8 \\
\hline 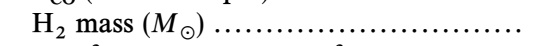 & $1900 \pm 700$ & $60 \pm 18$ & $18 \pm 6$ & $90 \pm 30$ & $60 \pm 18$ \\
\hline$n\left(10^{3} \mathrm{H}_{2}\right.$ molecules $\left.\mathrm{cm}^{-3}\right) \ldots \ldots \ldots \ldots \ldots$ & $5-10$ & $1-2$ & $0.8-1.7$ & $0.5-1.0$ & $0.6-1.0$ \\
\hline
\end{tabular}


densities for cloud $\mathrm{A}$ (expressed as $\mathrm{H}_{2}$ molecules $\mathrm{cm}^{-3}$ ) were estimated by taking into account that the molecular material is distributed in two relatively large clumps, corresponding to both the $\mathrm{E}$ and $\mathrm{W}$ clumps, $1^{\prime} .9 \times 0.7$ and $0^{\prime} .8$ $\times 0.2$ in size, respectively, and a number of unresolved clumps $(N \approx 30), 18^{\prime \prime}$ in diameter each (comparable densities are derived if $N=10-100$ ). (A HPBW $=22^{\prime \prime}$ corresponds to $0.48 d_{5 \mathrm{kpc}}$.) Molecular masses and volume densities corresponding to $d=5.0,3.7$, and $6.3 \mathrm{kpc}$ are listed in the table. Spherical clumps were assumed for clouds B-E. The presence of clumps is clear in other molecular clouds (see, e.g., Koo 1999) and in regions exposed to a strong UV radiation field.

As is seen from the table, the bulk of the molecular material $\left(\simeq 1200 \pm 500 d_{5 \mathrm{kpc}}^{2} M_{\odot}\right)$ appears related to the southern bar (cloud A), while little molecular gas $\left(\simeq 140 \pm 50 d_{5 \mathrm{kpc}}^{2} M_{\odot}\right)$ has been found to be associated with the W-R windblown bubble (clouds B-E). The derived density for cloud A agrees, to within errors, with previous estimates by SHWB and St-Louis et al. (1998).

It is worth noting that the molecular mass derived in this paper for the component at $54 \mathrm{~km} \mathrm{~s}^{-1}$ is larger than the determinations by SHWB and Rizzo et al. (2001). Regarding the SHWB paper, the difference arises in the fact that these more sensitive and higher angular resolution observations have enabled us to identify individual clumps and to detect $\mathrm{CO}$ emission in a larger area. Rizzo et al.'s estimates were derived applying LVG diagrams, and the differences may show the uncertainties involved in the different methods.

\subsubsection{PDR at the Interface Region between the Ionized and the} Molecular Gas

Figure 5 displays an overlay of the neutral hydrogen distribution within the velocity range of $51.4-57.9 \mathrm{~km} \mathrm{~s}^{-1}$, as obtained with the VLA by CGNO (white contours), the inte-

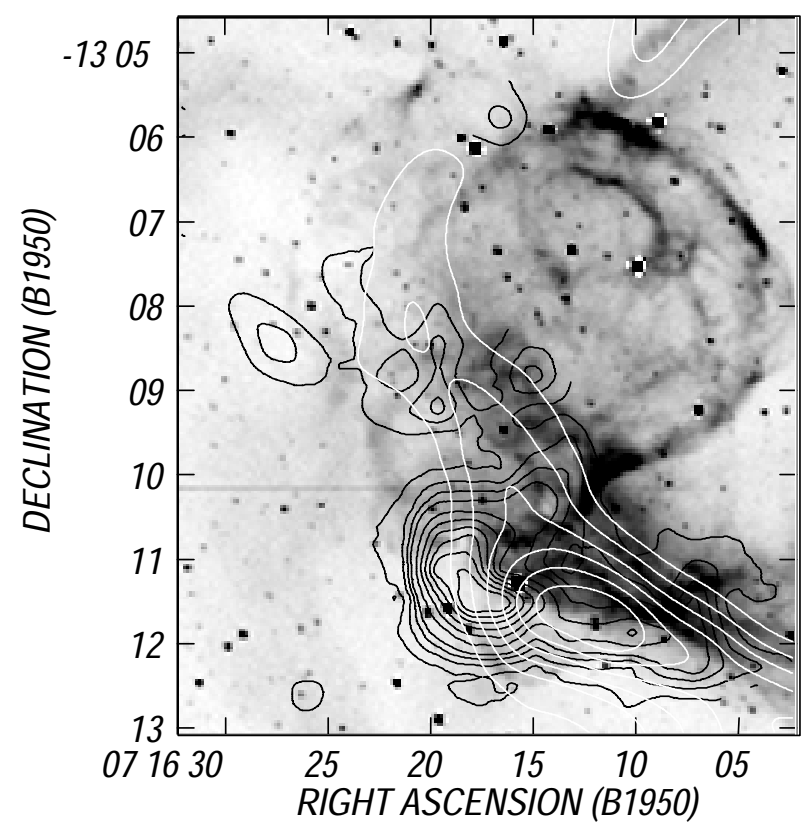

FIG. 5.-Overlay of the integrated $\mathrm{H}$ I emission within the velocity interval 51.4-57.9 $\mathrm{km} \mathrm{s}^{-1}$ (white contours) (CGNO), the integrated CO (2-1) line emission corresponding to the molecular component at $54 \mathrm{~km}$ $\mathrm{s}^{-1}$ (black contours) and the $\mathrm{H} \alpha$ image of the nebula (gray scale). The $\mathrm{H} \mathrm{I}$ contour lines correspond to $5,15,25,35,45$, and 55 mJy beam $^{-1}$. The CO contours are the same as in Fig. 2. grated $\mathrm{CO}$ emission (black contour lines), and the $\mathrm{H} \alpha$ image (gray scale). The main H I clump at $\alpha=7^{\mathrm{h}} 16^{\mathrm{m}} 12^{\mathrm{s}}, \delta=$ $-13^{\circ} 11^{\prime} 7$ (B1950.0) appears to be projected onto the gap between the clumps in cloud $A$, suggesting that the $\mathrm{H}_{2}$ material has been dissociated in this region. Clouds B and $\mathrm{D}$ are projected onto a region of weak $\mathrm{H}$ I emission, while cloud $\mathrm{C}$ is detected where the $\mathrm{H}$ I filament is incomplete. The neutral atomic mass in the $\mathrm{H}$ I filament at $54 \mathrm{~km} \mathrm{~s}^{-1}$ $\left(50 d_{5 \mathrm{kpc}}^{2} M_{\odot}, \mathrm{CGNO}\right)$ is only a small fraction of the total molecular mass.

In spite of the different angular resolutions, the distribution of the different gas components supports the idea of the $\mathrm{H}$ I filament being placed at the interface between the ionized and molecular gas. Thus, the $\mathrm{H}$ I filament at $54 \mathrm{~km}$ $\mathrm{s}^{-1}$ seems to have originated in the dissociation of the molecular gas. Although the action of shock fronts cannot be discarded, the presence of the ionization front indicates that a PDR has developed at the surface of the molecular cloud, illuminated by far-UV radiation (see Hollenbach \& Tielens 1997).

St-Louis et al. (1998) performed narrowband $\mathrm{H}_{2}$ infrared observations centered on the $v=1-0 \mathrm{~S}(1)$ transition. They found that the $\mathrm{H}_{2}$ emission is filamentary and lies mainly on the southern border of the bar or in regions of very low optical emission. Their Figure 1 shows that the brightest $\mathrm{H}_{2}$ filaments follow the southern border of the bar from $\alpha=7^{\mathrm{h}} 16^{\mathrm{m}} 3^{\mathrm{s}}$ to $7^{\mathrm{h}} 16^{\mathrm{m}} 12^{\mathrm{s}}$. This region is placed at the interface between the ionized and $\mathrm{CO}$ emission features (see Fig. 2). Thus, the excited $\mathrm{H}_{2}$ emission probably resides on the molecular cloud's surface, as expected in PDRs (see, e.g., Levenson et al. 2000). The $\mathrm{H}_{2}$ emission also coincides with the brightest region of the main CO clump. This correlation between $\mathrm{H}_{2}$ and $\mathrm{CO}$ lines is also expected in PDRs (Rubio et al. 2000).

Additional support for the PDR hypothesis comes from the relatively large $R_{21}$ values derived for the W clump and the molecular material projected onto the bar. Sakamoto et al. (1994) have found comparable line ratios in regions of the Orion molecular clouds, which are exposed to a strong FUV radiation field.

In an attempt to look for signs of star formation, we have searched for protostellar candidates in the IRAS Point Source Catalog. The IRAS point source 07161-1311 $\left(\alpha=7^{\mathrm{h}} 16^{\mathrm{m}} 12^{\mathrm{s}}, \delta=-13^{\circ} 11^{\prime} 7\right.$ [B1950.0]) is seen projected onto the molecular cloud. The source fulfills the conditions $1.2 \leq F(100 \mu \mathrm{m}) / F(60 \mu \mathrm{m}) \leq 6$ and $F(60 \mu \mathrm{m}) / F(25 \mu \mathrm{m}) \geq 1$, which discriminate against cold IRAS point sources (Junkes, Fürst, \& Reich 1992). However, the flux density ratios, $\log [F(12 \mu \mathrm{m}) / F(25 \mu \mathrm{m})]=-0.26$ and $\log [F(25$ $\mu \mathrm{m}) / F(60 \mu \mathrm{m})]=-1.4$, are consistent with a hot cirrus heated by UV photons from nearby massive stars (Yamaguchi et al. 1999), suggesting that the IR source is not a protostellar object.

The appearance of cloud A is that of a molecular cloud bounded by the ionization front viewed edge-on, with the molecular material being disrupted by the energetic flux of the star. The morphology of cloud A, which encircles the bar, suggests the presence of a secondary bubble, which might be colliding with the main W-R filamentary bubble (see SHWB; St-Louis et al. 1998).

\subsection{Molecular Clouds at 37 and $67 \mathrm{~km} \mathrm{~s}^{-1}$}

The integrated CO (2-1) line emission corresponding to the molecular clouds at 37 and $67 \mathrm{~km} \mathrm{~s}^{-1}$ is shown in 
Figures 6 and 7, respectively, superposed on the $\mathrm{H} \alpha$ image of the nebula. The cloud at $37 \mathrm{~km} \mathrm{~s}^{-1}$ appears projected onto a region of high optical absorption to the east of the filamentary bubble. The western boundary of the molecular cloud follows the eastern border of the filamentary bubble and the rim of a small optical ring near $\alpha=7^{\mathrm{h}} 16^{\mathrm{m}} 22^{\mathrm{s}}, \delta=$ $-13^{\circ} 9^{\prime} .0$. The molecular cloud extends to the east and north beyond the observed area. No H I gas with velocities close to $37 \mathrm{~km} \mathrm{~s}^{-1}$ was detected in the VLA data (see Fig. 4 in the

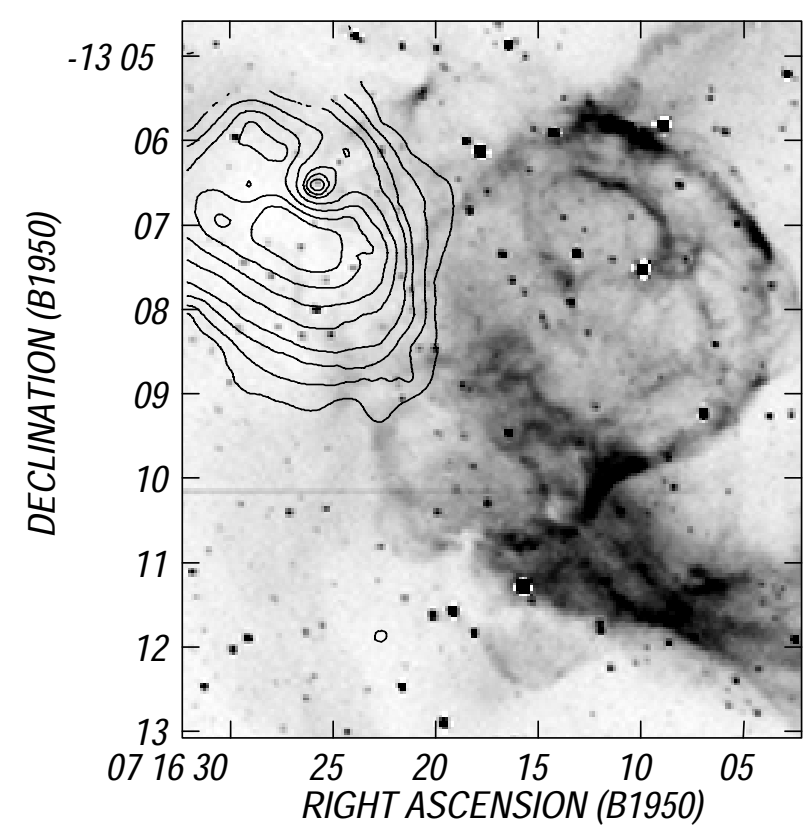

FIG. 6.- $\mathrm{CO}$ integrated emission for the component at $37 \mathrm{~km} \mathrm{~s}^{-1}$ superposed on the $\mathrm{H} \alpha$ image of the nebula. The contour lines are 0.7, 1.2, $1.8,2.4,3.6,4.8,6.0,7.2$, and $8.4 \mathrm{~K} \mathrm{~km} \mathrm{~s}^{-1}$.

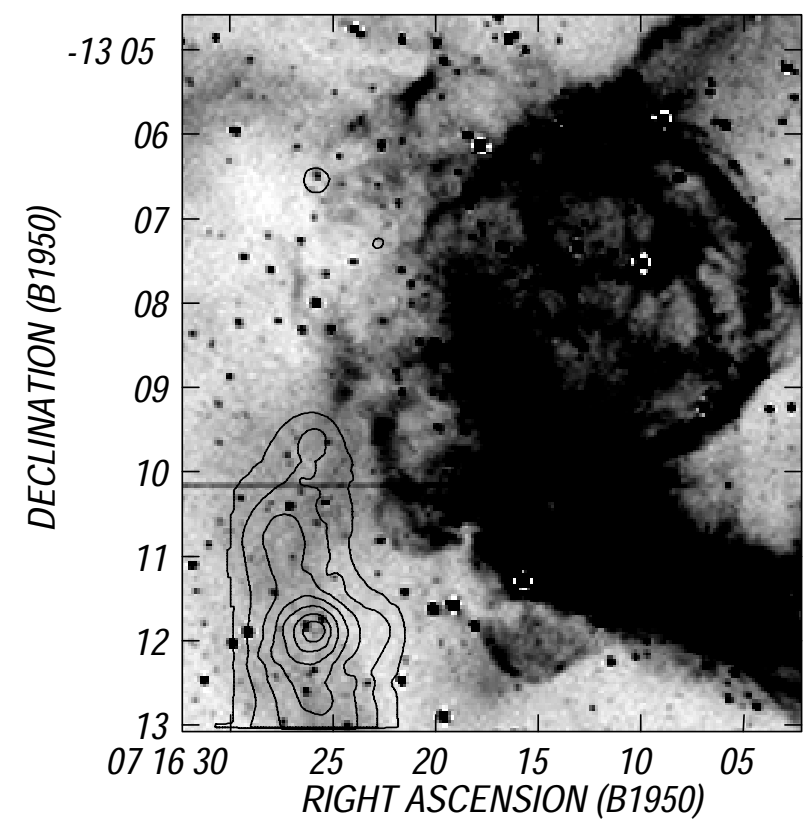

FIG. 7.-Same as Fig. 6 , but for $67 \mathrm{~km} \mathrm{~s}^{-1}$. The contour lines are from 0.7 to $3.7 \mathrm{~K} \mathrm{~km} \mathrm{~s}^{-1}$ in intervals of $0.5 \mathrm{~K} \mathrm{~km} \mathrm{~s}^{-1}$. paper by CGNO). The $\mathrm{H}$ I filament at $54 \mathrm{~km} \mathrm{~s}^{-1}$ appears projected onto the western boundary of the molecular cloud, suggesting a possible interaction. However, from the present observational data, it is not clear if the molecular material is interacting with the nebula.

The cloud at $67 \mathrm{~km} \mathrm{~s}^{-1}$ was detected to the southeast of NGC 2359 and is projected onto one of the southern optical streamers. The comparison between the molecular emission at $67 \mathrm{~km} \mathrm{~s}^{-1}$ and the $\mathrm{H} \mathrm{I}$ emission distribution at the same velocity (see Fig. 4; CGNO) suggests that both gas components are complementary. This molecular material appears to be unconnected with the nebula.

Line widths of the spectra, corresponding to the molecular clouds at 37 and $67 \mathrm{~km} \mathrm{~s}^{-1}$, differ significantly from line widths in the cloud at $54 \mathrm{~km} \mathrm{~s}^{-1}$. The profiles at 37 and 67 $\mathrm{km} \mathrm{s}^{-1}$ are narrow, with FWHMs in the range of 0.9-2.0 $\mathrm{km} \mathrm{s}^{-1}$ (Fig. $4 e$ and $4 f$ ). From one position observed in ${ }^{12} \mathrm{CO}$ and ${ }^{13} \mathrm{CO}$ lines in the cloud at $37 \mathrm{~km} \mathrm{~s}^{-1}$ (Fig. 4e), we estimate $T_{\text {exc }}=12 \mathrm{~K}, \tau\left({ }^{13} \mathrm{CO}\right)=0.4$ and $\tau\left({ }^{12} \mathrm{CO}\right)=13$. Here $R_{21}$ values corresponding to these clouds are about 0.5-0.6.

The $\mathrm{H}_{2}$ column densities, $\mathrm{CO}$ luminosities and the molecular masses are $(10 \pm 4) \times 10^{20} \mathrm{~cm}^{-2}, 69.7 d_{5 \mathrm{kpc}}^{2} \mathrm{~K} \mathrm{~km} \mathrm{~s}^{-1}$ $\mathrm{pc}^{2}$ and $380 \pm 120 d_{5 \mathrm{kpc}}^{2} M_{\odot}$ for the cloud at $37 \mathrm{~km} \mathrm{~s}^{-1}$ and $(5 \pm 2) \times 10^{20} \mathrm{~cm}^{-2}, 12.8 d_{5 \mathrm{kpc}}^{2} \mathrm{~K} \mathrm{~km} \mathrm{~s}^{-1} \mathrm{pc}^{2}$ and 70 $\pm 25 d_{5 \mathrm{kpc}}^{2} M_{\odot}$ for the cloud at $67 \mathrm{~km} \mathrm{~s}^{-1}$. The $\mathrm{H}_{2}$ column densities and molecular masses were derived as described in $\S 3.1$.

\section{ENERGETICS OF NGC 2359 AND THE NATURE OF THE MOLECULAR GAS}

Several authors have analyzed the energetics of NGC 2359. Treffers \& Chu (1982), taking into account only the ionized material, have estimated that the kinetic energy in the shell is less than $1 \%$ of the kinetic energy of the stellar wind, suggesting that the nebula is in the momentumconserving stage. The analysis by Marston (1991), who included the neutral mass resulting from the dust mass and the normal gas-to-dust mass ratio, also provides a similar result. CGNO, taking into account the ionized and neutral mass derived from VLA observations and the molecular mass obtained by SHWB, concluded that the nebula was in the momentum-conserving stage or in an intermediate stage between energy and momentum conservation. According to Treffers \& Chu, strong radiative losses can occur when neutral clumps remain immersed within the bubble (see also Hartquist \& Dyson 1993).

Following CGNO, we have reanalyzed the energetics of the (1) filamentary shell and (2) the entire complex separately by taking into account these new molecular results. We derive the kinetic efficiency $\epsilon$ as the ratio between the kinetic energy of the swept-up mass $\left(E_{k}\right)$ and the mechanical energy released to the interstellar medium (ISM) by the stellar wind $\left(E_{w}\right)$.

1. We consider $70 d_{5 \mathrm{kpc}}^{2} M_{\odot}$ of ionized material, $25 d_{5 \mathrm{kpc}}^{2}$ $M_{\odot}$ of neutral atomic gas (half the mass of the filament associated with the filamentary bubble and with the southern bar), and $140 d_{5 \mathrm{kpc}}^{2} M_{\odot}$ of molecular material to be associated with the filamentary bubble. For expansion velocities in the range of $15-30 \mathrm{~km} \mathrm{~s}^{-1}$ (see CGNO), $E_{k}=$ $0.5-2.1 \times 10^{48} d_{5 \mathrm{kpc}}^{2}$ ergs. Adopting $E_{w} \simeq 0.5-1.0 \times 10^{50}$ ergs as the mechanical energy released into the ISM by the W-R star during the dynamical age of the WBB (CGNO), $\epsilon \simeq 0.005-0.04 d_{5 \mathrm{kpc}}^{2}$. These values for the kinetic efficiency 
suggest that the filamentary shell is in the momentumconserving stage or in an intermediate stage between energy and momentum conservation. Similar $\epsilon$ values are derived for distances in the range of $3.7-6.3 \mathrm{kpc}$.

2 . We evaluate $E_{k}$ for the whole NGC 2359 complex by including in our calculations the ionized and neutral gas in the bar and streamers. The ionized material in these regions amounts to $190-400 d_{5 \mathrm{kpc}}^{2} M_{\odot}$, the neutral atomic gas detected within the velocity range of $41-63 \mathrm{~km} \mathrm{~s}^{-1}$ is about $300 d_{5 \mathrm{kpc}}^{2} M_{\odot}$, and the molecular material at $54 \mathrm{~km} \mathrm{~s}^{-1}$ is $1200 \pm 500 d_{5 \mathrm{kpc}}^{2} M_{\odot}$. Assuming this material is expanding at $6 \mathrm{~km} \mathrm{~s}^{-1}$ (see CGNO) and taking into account the kinetic energy of the filamentary bubble derived in point $1, E_{k} \simeq$ $0.9-2.6 \times 10^{48} d_{5 \mathrm{kpc}}^{2}$ ergs. Adopting $E_{w} \simeq 1.6-3 \times 10^{50}$ ergs, which includes the energy released by the star during the $\mathrm{O}$ and current $\mathrm{W}-\mathrm{R}$ phases, $\epsilon \simeq 0.003-0.02 d_{5 \mathrm{kpc}}^{2}$, indicating a similar situation as in point 1 . No significant change in this conclusion follows if either distances in the range of $3.7-6.3 \mathrm{kpc}$ are considered or if the molecular material at $37 \mathrm{~km} \mathrm{~s}^{-1}$ is included.

In summary, our final result indicates that NGC 2359 is in the momentum-conserving stage or in an intermediate stage between energy and momentum conservation. This conclusion does not change if the lower masses derived by Rizzo el al. are considered.

The origin of the molecular material associated with NGC 2359 may shed some light on the formation of the nebula. Two possible origins can be considered for this gas: (1) it may predominantly consist of stellar material ejected by the star in previous evolutionary phases, or (2) it may be mostly composed of interstellar material. In this latter case, the molecular material would consist of the leftovers of the molecular cloud in which the W-R star was born. This molecular matter would have been partially swept up by the action of the stellar winds of the W-R star or its progenitor star.

For the case of NGC 2359, some support to the first interpretation comes from the observed ${ }^{12} \mathrm{CO} /{ }^{13} \mathrm{CO}$ ratio. The ${ }^{12} \mathrm{C} /{ }^{13} \mathrm{C}$ isotopic abundances can be estimated whenever the ${ }^{12} \mathrm{CO} /{ }^{13} \mathrm{CO}$ ratio reflects the ${ }^{12} \mathrm{C} /{ }^{13} \mathrm{C}$ isotopic ratio. The isotopic abundance is $\approx 70$ in the local ISM (Wilson \& Matteucci 1992), while values in the range of 10-50 were derived for planetary nebulae and the envelopes of asymptotic giant branch stars (see, e.g., Bachiller et al. 1997). These lower ratios are the result of stellar processing. As mentioned in $\S 3.1 .1, W\left({ }^{12} \mathrm{CO}\right) / W\left({ }^{13} \mathrm{CO}\right) \simeq 8-11$ for the three points in the direction of the E clump for which ${ }^{13} \mathrm{CO}$ observations are available. However, the ${ }^{12} \mathrm{CO}$ emission should be optically thin so that the ${ }^{12} \mathrm{CO} /{ }^{13} \mathrm{CO}$ ratio reflects the isotopic abundance. We do not know if this is the case, since the ${ }^{13} \mathrm{CO}$ observations were obtained in the direction of the brightest parts of the molecular clump.

The large molecular mass found to be associated with the nebula casts more doubt on the first interpretation. For the filamentary shell alone, the molecular mass amounts to $\simeq 140 d_{5 \mathrm{kpc}}^{2} M_{\odot}$, indicating that the material is interstellar rather than stellar in origin. If we adopt the lower limit to the photometric distance $(d=2.8 \mathrm{kpc}$, see $\S 3)$, the molecular mass amounts to $\geq 40 M_{\odot}$, suggesting an interstellar origin for most of the gas. Taking into account the ionized, neutral, and molecular mass, $\simeq 240 d_{5 \mathrm{kpc}}^{2} M_{\odot}$ are related to the filamentary WBB. To the contrary, the small molecular mass found to be connected with the ring nebulae around W-R 16 and 75 (Marston et al. 1999a, 1999b) is compatible with a stellar ejecta origin. Regarding the molecular material related to the southern bar, the amount of molecular gas indicates an interstellar origin.

\section{SUMMARY}

We have investigated the distribution of the molecular material related to NGC 2359, based on CO (2-1) observations carried out with the SEST with an angular resolution of $22^{\prime \prime}$. These new observations, obtained with higher angular resolution and sensitivity than the previous observations by SHWB, enabled us to image the molecular material in the environs of the nebula. Three molecular components at 37,54 , and $67 \mathrm{~km} \mathrm{~s}^{-1}$ were observed in direction of NGC 2359. Most of the molecular gas associated with the nebula is detected at $54 \mathrm{~km} \mathrm{~s}^{-1}$, following the southern border of the bar. An $\mathrm{H}_{2}$ mass of about 1200 $\pm 500 d_{5 \mathrm{kpc}}^{2} M_{\odot}$ appears to be related to the bar, while $140 \pm 50 d_{5 \mathrm{kpc}}^{2} M_{\odot}$ can be linked to the filamentary bubble. Volume densities are $\geq 10^{3} \mathrm{H}_{2}$ molecules $\mathrm{cm}^{-3}$ for the molecular gas associated with the southern bar, while lower values were derived for the material connected with the filamentary WBB. The $\mathrm{H}_{2}$ mass in the components at 37 and $67 \mathrm{~km} \mathrm{~s}^{-1}$ are $380 \pm 120 d_{5 \mathrm{kpc}}^{2}$ and $70 \pm 25 d_{5 \mathrm{kpc}}^{2} M_{\odot}$. The association of the component at $37 \mathrm{~km} \mathrm{~s}^{-1}$ remains an open question, while the component at $67 \mathrm{~km} \mathrm{~s}^{-1}$ is most probably unrelated to the complex. Although part of the molecular gas associated with NGC 2359 may have originated in previous evolutionary phases of the current W-R star, it seems that this molecular gas is mostly composed by interstellar matter.

The comparison between the distributions of the ionized gas, the $\mathrm{H}$ I filament detected with the VLA at $54 \mathrm{~km} \mathrm{~s}^{-1}$, and the molecular gas at the same velocity suggests that the $\mathrm{H}$ I filament is located at the interface between the ionized and molecular material. We concluded that the $\mathrm{H}$ I filament at this velocity $\left(\simeq 50 M_{\odot}\right)$ has originated in the photodissociation of the molecular material. The relatively large values derived for the ${ }^{12} \mathrm{CO}(2-1) /{ }^{12} \mathrm{CO}(1-0)$ intensity ratio and the presence of excited $\mathrm{H}_{2}$ emission are consistent with the existence of a PDR at the surface of the molecular clouds. Thus, the presence of a PDR gives a natural explanation for the existence of the $\mathrm{H}$ I filament. The reanalysis of the dynamics of NGC 2359, taking into account the new $\mathrm{CO}$ results, confirms that the nebula is closer to the momentum-conserving stage.

More studies of W-R ring nebulae, including the ionized, neutral, and molecular gas components, are necessary to confirm if a similar dynamical situation applies to other ring nebulae and to investigate the origin of the molecular material in their environs. This is important to improve theoretical models of evolving interstellar bubbles, which might enable the parameters of the stars to be estimated based on their actions on the surrounding ISM. Additional studies of the PDR at the interface between the ionized and the molecular material, using molecular lines that can trace higher density regions, are also required.

We very much acknowledge the anonymous referee, whose comments and suggestions lead to the improvement of this paper. C. E. C. and M. R. are grateful to the director and staff of the SEST for the support received during the observations. C. E. C. acknowledges F. Bareilles for his help with one of the figures. The National Radio Astronomy 
Observatory is a facility of the National Science Foundation operated under cooperative agreement by Associated Universities, Inc. C. C. acknowledges support from Facultad de Ciencias Astronómicas y Geofísicas, Universidad
Nacional de La Plata, project G037 (UNLP), and CONICET project PIP 607/98, Argentina. M. R. acknowledges support from FONDECYT (Chile) through grants 1990881 and 79900042.
Bachiller, R., Forveille, T., Huggins, P. J., \& Cox, P. 1997, A\&A, 324, 1123

Booth, R., et al. 1989, A\&A, 216, 315

Brand, J., \& Blitz, L. 1993, A\&A, 275, 67

Burton, W. B. 1988, in Galactic and Extragalactic Radioastronomy, ed.

G. L. Verschuur \& K. I. Kellerman (2d ed.; New York: Springer), 295

Cappa, C. E., Goss, W. M., Niemela, V. S., \& Ostrov, P. 1999, AJ, 118, 948 (CGNO)

Castets, A., Duvert, G., Dutrey, A., Bally, J., Langer, W. D., \& Wilson, R. W. 1990, A\&A, 234, 469

Chu, Y.-H. 1981, ApJ, 249, 195

Conti, P. S., \& Vacca, W. D. 1990, AJ, 100, 431

Digel, S. W., Grenier, I. A., Heithausen, A., Hunter, S. D., \& Thaddeus, P. 1996, ApJ, 463, 609

Goudis, C., Hippelun, H., \& Münch, G. 1983, A\&A, 117, 127

Hartquist, T. W., \& Dyson, J. E. 1993, QJRAS, 34, 57

Hollenbach, D. J., \& Tielens, A. G. G. M. 1997, ARA\&A, 35, 179

Johnson, H. M., \& Hogg, D. E. 1965, ApJ, 142, 1033

Junkes, N., Fürst, E., \& Reich, W. 1992, A\&A, 261, 289

Koo, B.-C. 1999, ApJ, 518, 760

Levenson, N. A., et al. 2000, ApJ, 533, L53

Marston, A. P. 1991, ApJ, 366, 181

\section{REFERENCES}

Marston, A. P., Welzmiller, J., Bransford, M. A., Black, J. H., \& Bergman, P. 1999a, ApJ, 518, 769

. 1999b, in IAU Symp. 193, Wolf-Rayet Phenomena in Massive Stars and Starburst Galaxies, ed. K. A. van der Hucht, G. Koenigsberger, \& P. R. J. Eenens (San Francisco: ASP), 370

Rizzo, J. R., Martin-Pintado, J., \& Mangum, J. G. 2001, A\&A, 366, 146

Rubio, M., Contursi, A., Lequeux, J., Probst, R., Barbá, R., Boulanger, F., Cesarsky, D., \& Maoli, R. 2000, A\&A, 359, 1139

Sakamoto, S., Hayashi, M., Hasegawa, T., Handa, T., \& Oka, T. 1994, ApJ, 425,641

Schneps, M. H., Haschick, A. D., Wright, E. L., \& Barrett, A. H. 1981, ApJ, 243, 184 (SHWB)

Smith, L. F., Shara, M. M., \& Moffat, A. F. J. 1996, MNRAS, 281, 163

St-Louis, N., Doyon, R., Chagnon, F., \& Nadeau, D. 1998, AJ, 115, 2475

Treffers, R. R., \& Chu, Y.-H. 1982, ApJ, 254, 569

van der Hucht, K. A. 2001, NewA Rev., 45, 135

van der Hucht, K. A., Hidayat, B., Admiranto, A. G., Supelli, K. R., \& Doom, C. 1988, A\&A, 199, 217

Wilson, T. L., \& Matteucci, F. 1992, A\&A Rev., 4, 1

Yamaguchi, R., Saito, H., Mizuno, N., Mine, Y., Mizuno, A., Ogawa, H., \& Fukui, Y. 1999, PASJ, 51, 791 